\title{
TEORES FOLIARES DE NUTRIENTES EM GENÓTIPOS DE BANANEIRA ${ }^{1}$
}

\author{
ANA LÚCIA BORGES², SEBASTIÃO DE OLIVEIRA E SILVA², RANULFO CORRÊA CALDAS², \\ CARLOS ALBERTO DA SILVA LEDO²
}

\begin{abstract}
RESUMO - A diagnose foliar é bastante útil na determinação do estado nutricional das plantas. As exigências nutricionais da bananeira podem diferir em função das suas características genéticas. O trabalho objetivou determinar os teores de macro e micronutrientes na terceira folha de genótipos de bananeira. Foram selecionados 24 genótipos de bananeira (triplóides e tetraplóides), em dois ciclos de produção (1999 e 2000), do Banco Ativo de Germoplasma de Banana da Embrapa Mandioca e Fruticultura Tropical, determinando-se os teores foliares de macronutrientes $(\mathrm{N}, \mathrm{P}, \mathrm{K}, \mathrm{Ca}, \mathrm{Mg}$ e S) e de micronutrientes (B, Cl, Cu, Fe, Mn e Zn). Os resultados indicaram que existe variação nos teores foliares entre plantas do mesmo grupo genômico e entre os ciclos de produção, com teores médios mais elevados no segundo ciclo para N, $\mathrm{P}, \mathrm{Ca}, \mathrm{Mg}, \mathrm{S}, \mathrm{Cu}$ e $\mathrm{Mn}$ e mais baixos para K, Cl, B, Fe e Zn. O N variou de 21,6 a 28,5 $\mathrm{g} \mathrm{kg}^{-1}$, o K de 13,7 a 30,8 g kg-1 e foram os macronutrientes com teores mais elevados nas folhas. O teor de $\mathrm{Cl}$ variou de 10,4 a 24,7 $\mathrm{g} \mathrm{kg}^{-1}$, o Mn de 43 a $574 \mathrm{mg} \mathrm{kg}^{-1}$ e o Fe de 56 a $212 \mathrm{mg} \mathrm{kg}^{-1}$, sendo os micronutrientes com teores mais elevados nas folhas.
\end{abstract}

Termos para indexação: Musa spp., triplóides, tetraplóides, macronutrientes, micronutrientes.

\section{LEAF NUTRIENTS CONTENTS IN BANANA GENOTYPES}

\begin{abstract}
The leaf diagnosis is quite useful for the determination of the nutritional state of plants. The nutritional banana tree demands can differ in accordance with genetic characteristics. The aim of present work was to determinate the macro and micronutrients contents in the third leaf of banana genotypes. Twenty four banana genotypes were selected (triploids and tetraploids), in two production cycles (1999 and 2000), in the Active Germoplasm Bank of Banana at Embrapa Mandioca e Fruticultura Tropical, in Cruz das Almas, Bahia, Brazil. The leaf contents of macronutrients (N, P, K, Ca, Mg and S) and micronutrients (B, Cl, Cu, Fe, Mn and $\mathrm{Zn}$ ) were determined. The results showed that occurred variation in leaf contents among plants in the same genome group and between the first and second production cycles, with higher medium levels in the second cycle for $\mathrm{N}, \mathrm{P}, \mathrm{Ca}, \mathrm{Mg}, \mathrm{S}, \mathrm{Cu}$ and $\mathrm{Mn}$ and lower ones for $\mathrm{K}, \mathrm{Cl}, \mathrm{B}, \mathrm{Fe}$ and $\mathrm{Zn}$. The nitrogen contents 21.6 to $28.5 \mathrm{~g} \mathrm{~kg}^{-1}$ and $\mathrm{K} 13.7$ to $30.8 \mathrm{~g} \mathrm{~kg}^{-1}$ and they were the macronutrients with the highest contents in the leaves. The chlorine contents was 10.4 to $24.7 \mathrm{~g} \mathrm{~kg}^{-1}$, manganese 43 to $574 \mathrm{mg} \mathrm{kg}^{-1}$ and iron 56 to $212 \mathrm{mg} \mathrm{kg}^{-1}$, and they were the micronutrients with the highest contents in leaves.
\end{abstract}

Index terms: Musa spp., triploids, tetraploids, macronutrients, micronutrients.

\section{INTRODUÇÃO}

A bananeira (Musa spp.), originária do Continente Asiático, é cultivada em quase todos os países tropicais. No Brasil, segundo produtor mundial de banana, totalizando em 2004, aproximadamente, 6,6 milhões de toneladas, a cultura é praticada de norte a sul do País.

É uma planta exigente em nutrientes, principalmente o nitrogênio $(\mathrm{N})$ e o potássio $(\mathrm{K})$, sendo este o nutriente mais absorvido (Faria, 1997). Em média, a bananeira absorve $16 \mathrm{~kg}$ de $\mathrm{K}$ por tonelada de frutos produzidos, sendo que a 'Prata-Anã' extrai $21,5 \mathrm{~kg} \mathrm{t}^{-1} \mathrm{de}$ frutos (Faria, 1997), enquanto a 'Pacovan' absorve 21,7 $\mathrm{kg} \mathrm{t}^{-1}$ de frutos (Neves et al., 1991). Apesar de ambas as variedades produzirem frutos tipo Prata, tem-se observado diferenças entre elas quanto à absorção de nutrientes (Faria, 1997).

A diagnose foliar vem sendo bastante útil na quantificação do estado nutricional das culturas e nas recomendações de adubação (Walsh \& Beaton, 1973), no qual o teor do nutriente na planta é resultante da ação e interação dos fatores que afetam a sua disponibilidade no solo e absorção pela planta (Munson \& Nelson, 1973). A avaliação do estado nutricional das culturas pela diagnose foliar analisa determinadas folhas em períodos definidos da vida da planta, pois as folhas, de maneira geral, são os órgãos que refletem melhor o estado nutricional, isto é, respondem mais a variações no suprimento do nutriente (Malavolta et al., 1989).

Para a interpretação dos resultados da análise foliar, estabeleceram-se teores padrões, baseando-se na correlação entre a concentração de nutrientes nas folhas e o desenvolvimento ou produtividade da cultura. Os teores padrões de referência não têm aplicação universal, pois estão relacionados com fatores climáticos, solo e cultivares. Assim, os valores de referência quase sempre precisam ser adaptados às condições locais, embora alguma extrapolação sempre seja possível (Bataglia \& Dechen, 1986).
Quanto aos teores padrões para bananeira, segundo IFA (1992), os níveis ótimos padronizados para a terceira folha em cultivares do subgrupo Cavendish, com a inflorescência totalmente aberta, são, em $\mathrm{g} \mathrm{kg}^{-1}$ : 27,0-36,0 de N; 1,6-2, 7 de P; 32,0-54,0 de K; 6,6-12,0 de Ca; 2,7-6,0 de $\mathrm{Mg}$; 1,6-3,0 de Se 9,0-18,0 de Cl, e em mg kg-1: 80-360 de Fe, 200-1.800 de Mn; 20-50 de Zn; 10-25 de B e 6-30 de Cu. Silva et al. (2002) estabeleceram faixas de suficiência para bananeira 'Prata-Anã' no norte de Minas Gerais, sendo, em g kg-1: 25,0-29,0 de N; 1,5-1,9 de P; 27,0-35,0 de K; 4,5-7,5 de Ca; 2,4-4,0 de Mg; 1,7-2,0 de S, e em mg $\mathrm{kg}^{-1}: 12,0-25,0$ de B; 2,6-8,8 de Cu; 72,0-157,0 de Fe; 173,0-630,0 de Mn e 14,0-25,0 de Zn. Para 'Pacovan', em Petrolina (PE), Borges \& Caldas (2004) definiram as faixas, em $\mathrm{g} \mathrm{kg}^{-1}: 22,0-24,0 \mathrm{de} \mathrm{N} ; 1,7-1,9 \mathrm{de} \mathrm{P} ; 25,0-$ 28,0 de K; 6,3-7,3 de Ca; 3,1-3,5 de Mg; 1,7-1,9 de S, e em mg kg-1 : 1316 de B; 6-7 de $\mathrm{Cu} ; 71-86$ de Fe; $315-398$ de Mn e 12-14 de Zn.

O trabalho objetivou determinar os teores de macro e micronutrientes na terceira folha de 24 genótipos de bananeira.

\section{MATERIAL E MÉTODOS}

Para realização deste estudo, foram selecionados 24 genótipos de bananeira, no Banco Ativo de Germoplasma (BAG) de Banana da Embrapa Mandioca e Fruticultura Tropical, em dois ciclos de produção. Os genótipos avaliados foram agrupados em triplóide AAB (Pacovan, Prata Comum, Prata-Anã e Thap Maeo), triplóide AAA (Grande Naine, Caipira e Nam), tetraplóide AAAA (Calipso, Bucaneiro e Ambrósia) e tetraplóide AAAB (Pioneira, Maravilha, FHIA-18, Tropical, Prata-Caprichosa, Prata-Graúda, Prata-Garantida, ST12-31, Japira, PV42-129, Preciosa, Vitória, Pacovan Ken e PV 42-53). A produtividade média está expressa em tonelada por ciclo (número de dias do plantio à colheita do $1^{\circ}$ e $2^{\circ}$ ciclos) e por ano, onde se considerou o ciclo de 365 dias (Tabela 1).

O BAG está localizado na área experimental da Embrapa

\footnotetext{
${ }^{1}$ (Trabalho 200-2005). Recebido: 02-12-2005. Aceito para publicação: 18-04-2006.

${ }^{2}$ Engenheiro(a) Agrônomo(a), Pesquisador(a) da Embrapa Mandioca e Fruticultura Tropical. Caixa Postal 007. CEP 44380-000, Cruz das Almas-BA. analucia@cnpmf.embrapa.br, ledo@cnpmf.embrapa.br.
} 
TABELA 1 - Características e produtividades médias dos 24 genótipos de bananeiras selecionados em dois ciclos de produção, nos anos de 1999-2000, Cruz das Almas-BA.

\begin{tabular}{|c|c|c|c|c|c|c|}
\hline \multirow{3}{*}{ Genótipo } & \multirow{3}{*}{$\begin{array}{c}\text { Grupo } \\
\text { Genômico }\end{array}$} & \multirow{3}{*}{ Origem } & \multicolumn{4}{|c|}{ Produtividade } \\
\hline & & & $1^{\circ}$ ciclo & $2^{\circ}$ ciclo & $1^{\circ}$ ciclo & $2^{\circ}$ ciclo \\
\hline & & & \multicolumn{2}{|c|}{----- t ha ${ }^{-1}$ ciclo ------ } & \multicolumn{2}{|c|}{--- t ha $^{-1}$ ano $^{-1}$} \\
\hline Pacovan & AAB & - & 21,4 & 26,5 & 20,1 & 29,6 \\
\hline Japira & AAAB & Pacovan & 23,2 & 31,1 & 22,7 & 29,8 \\
\hline PV42-129 & $\mathrm{AAAB}$ & Pacovan & 26,5 & 41,8 & 30,5 & 41,9 \\
\hline Preciosa & AAAB & Pacovan & 20,9 & 34,2 & 21,1 & 31,1 \\
\hline Vitória & $\mathrm{AAAB}$ & Pacovan & 25,6 & 49,4 & 24,5 & 41,5 \\
\hline Pacovan Ken & AAAB & Pacovan & 26,9 & 49,9 & 26,8 & 41,4 \\
\hline PV42-53 & $\mathrm{AAAB}$ & Pacovan & 27,5 & 33,9 & 27,4 & 30,8 \\
\hline Prata Comum & $\mathrm{AAB}$ & - & 12,7 & 19,7 & 12,3 & 18,5 \\
\hline Prata-Caprichosa & $\mathrm{AAAB}$ & Prata Comum & 28,5 & 30,3 & 29,9 & 29,3 \\
\hline Prata-Anã & AAB & - & 21,0 & 23,5 & 19,7 & 18,4 \\
\hline Pioneira & $\mathrm{AAAB}$ & Prata Anã & 11,8 & 22,7 & 13,1 & 25,7 \\
\hline Maravilha & $\mathrm{AAAB}$ & Prata Anã & 35,3 & 33,6 & 34,8 & 29,5 \\
\hline FHIA-18 & $\mathrm{AAAB}$ & Prata Anã & 25,1 & 36,9 & 26,8 & 37,7 \\
\hline Prata-Graúda & $\mathrm{AAAB}$ & Prata Anã & 34,1 & 35,2 & 34,5 & 29,5 \\
\hline Calipso & AAAA & High-Gate & 21,9 & 36,4 & 23,4 & 35,1 \\
\hline Bucaneiro & AAAA & High-Gate & 28,8 & 52,4 & 29,6 & 51,0 \\
\hline Ambrosia & AAAA & High-Gate & 23,7 & 54,7 & 24,8 & 56,2 \\
\hline Grande Naine & AAA & - & 36,4 & 37,1 & 36,8 & 33,0 \\
\hline Thap Maeo & $\mathrm{AAB}$ & - & 37,7 & 47,8 & 38,1 & 48,3 \\
\hline Caipira & AAA & - & 17,5 & 31,5 & 17,4 & 35,9 \\
\hline Nam & AAA & - & 16,6 & 31,7 & 17,1 & 33,2 \\
\hline Tropical & $\mathrm{AAAB}$ & Yangambi $n \stackrel{\circ}{2}$ & 19,1 & 35,7 & 18,5 & 35,6 \\
\hline Prata-Garantida & $\mathrm{AAAB}$ & Prata S. Tomé & 20,6 & 32,9 & 21,1 & 29,7 \\
\hline ST12-31 & $\mathrm{AAAB}$ & Prata São Tomé & 34,6 & 56,7 & 35,3 & 49,9 \\
\hline
\end{tabular}

Mandioca e Fruticultura Tropical, no Município de Cruz das Almas, Região do Recôncavo Baiano, a 1240'19" de latitude sul, 3906'22'” de longitude oeste Gr e altitude de $220 \mathrm{~m}$. O solo é um Latossolo Amarelo álico coeso franco-argiloarenoso (609 g de areia, $114 \mathrm{~g} \mathrm{de}$ silte e $277 \mathrm{~g}$ de argila por quilograma de solo), apresentando as propriedades químicas contidas na Tabela 2 . O clima da região é subúmido $\left(1.200 \mathrm{~mm}^{2} \mathrm{ano}^{-1}\right)$, com temperatura média anual de $24^{\circ} \mathrm{C}$ e umidade relativa do ar média anual de $80 \%$ (Souza \& Souza, 2001).

Durante os meses de março a maio, em 1999 (primeiro ciclo), e de abril a agosto, em 2000 (segundo ciclo), foram escolhidas aleatoriamente três plantas de cada genótipo, dentro do padrão varietal, e realizada amostragem da terceira folha a contar do ápice, com a inflorescência no estádio em que todas as pencas femininas se encontram descobertas (sem brácteas) e não mais de três pencas de flores masculinas (Martin-Prével, 1977). Essas amostras foram submetidas a análises químicas dos macronutrientes $(\mathrm{N}, \mathrm{P}, \mathrm{K}, \mathrm{Ca}, \mathrm{Mg}$ e S) e micronutrientes (B, Cu, Fe, Mn e $\mathrm{Zn}$ ), segundo metodologias propostas por Bataglia et al. (1983).

Além dos tratos culturais, como desbaste, desfolha e capinas, foi feita adubação a cada dois meses, manualmente, na quantidade de $100 \mathrm{~kg}_{\text {de }} \mathrm{N} \mathrm{ha}^{-1} \mathrm{ano}^{-1}$, na forma de uréia, $40 \mathrm{~kg}$ de $\mathrm{P}_{2} \mathrm{O}_{5}$ ha $^{-1} \mathrm{ano}^{-1}$, na forma de superfosfato simples e $300 \mathrm{~kg} \mathrm{de} \mathrm{K}_{2} \mathrm{O} \mathrm{ha}^{-1} \mathrm{ano}^{-1}$, na forma de $\mathrm{KCl}$. A suplementação de água foi feita quando necessária, por microaspersão.

Os dados dos teores de nutrientes nos dois ciclos de produção dos genótipos foram submetidos à análise de variância, no modelo inteiramente casualizado, usando procedimentos do SAS
(2005), e as médias estimadas, comparadas pelo teste de agrupamento de Scott \& Knott (Scott \& Knott, 1974), por meio do SAEG (2005).

\section{RESULTADOS E DISCUSSÃO}

Os teores médios dos macronutrientes apresentados na Tabela 3 indicaram valores mais elevados de N, P, Ca, Mg e S no segundo ciclo, para a maioria dos genótipos. Apenas os teores de $\mathrm{P}$ não diferiram entre os genótipos, nos dois ciclos de produção, possivelmente pela baixa absorção de P pela bananeira e a pequena amplitude da faixa de teor, quando se compara com os macronutrientes primários. $\mathrm{O}$ teor de $\mathrm{N}$ não variou no segundo ciclo, cujo valor médio foi de $25,6 \mathrm{~g} \mathrm{~kg}^{-1}$. No primeiro ciclo, observaram-se dois agrupamentos de genótipos, pelo teste de Scott \&Knott, para N (médias de25,3 e 22,9 $\mathrm{g} \mathrm{kg}^{-1}$ ) e Ca (médias de 9,9 e $6,5 \mathrm{~g} \mathrm{~kg}^{-1}$ ) e, no segundo ciclo, para $\mathrm{K}$ (médias de 25,0 e 16,7 $\mathrm{g} \mathrm{kg}^{-1}$ ), Ca (médias de 15,2 e 8,3 $\mathrm{g} \mathrm{kg}^{-1}$ ) e S (médias de 2,4 e 1,5 $\mathrm{g} \mathrm{kg}^{-1}$ ). Para o K (médias de 29,3; 24,1 e 19,0 $\mathrm{g} \mathrm{kg}^{-1}$ ), no primeiro ciclo, e $\mathrm{Mg}$ (médias de 7,2; 5,3 e 3,7 $\mathrm{g} \mathrm{kg}^{-1}$ ), no segundo ciclo, observam-se três agrupamentos, pelo teste de Scott \& Knott (Tabela 3). As maiores variações nos teores de nutrientes foram observadas no primeiro ciclo, para $\mathrm{Mg}$ (médias de 5,$5 ; 4,5 ; 3,5$ e 2,6 $\mathrm{g} \mathrm{kg}^{-1}$ ) e $\mathrm{S}$ (médias de 1,$8 ; 1,4 ; 1,2$ e $0,9 \mathrm{~g} \mathrm{~kg}^{-1}$ ), com quatro agrupamentos, apesar de não apresentar os menores coeficientes de variação (Tabela 3 ).

Os teores médios de N, P e K estão abaixo daqueles estabelecidos pelo IFA (1992), para variedades de banana do subgrupo Cavendish (AAA), sugerindo a necessidade de definir padrões para as condições e genótipos locais. Comparando a 'Prata-Anã' e seus

TABELA 2 - Propriedades químicas do solo nas profundidades de 0-20 e 20-40 cm da área experimental. Cruz das Almas-BA. 1999.

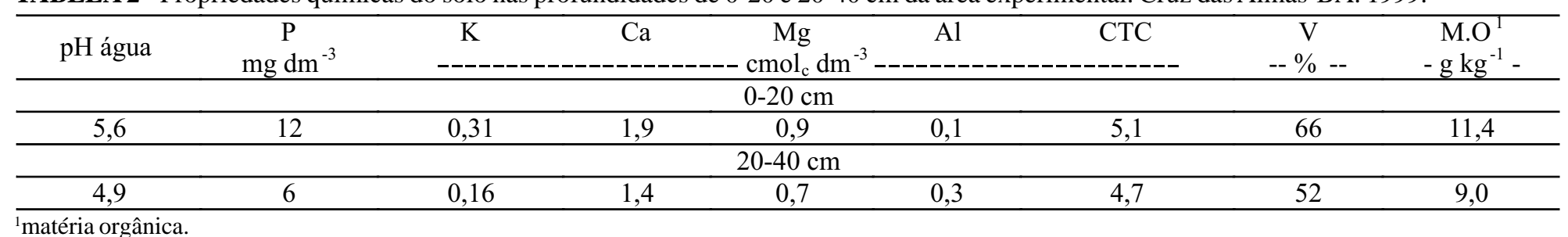


TABELA 3 - Teores foliares de macronutrientes em 24 genótipos de bananeira, no primeiro (1ํㅡ) e segundo ( $\left.2^{\circ}\right)$ ciclos de produção, nos anos de 1999-2000, Cruz das Almas-BA.

\begin{tabular}{|c|c|c|c|c|c|c|c|c|c|c|c|c|}
\hline \multirow[b]{2}{*}{ Genótipos } & \multicolumn{2}{|c|}{$\mathbf{N}$} & \multicolumn{2}{|c|}{$\mathbf{P}$} & \multicolumn{2}{|c|}{$\mathbf{K}$} & \multicolumn{2}{|c|}{$\mathbf{C a}$} & \multicolumn{2}{|c|}{ Mg } & \multicolumn{2}{|c|}{$\mathbf{S}$} \\
\hline & $1^{0}$ & $2^{-9}$ & $1^{0}$ & $2^{-0}$ & $1^{0}$ & $2^{-9}$ & $1^{0}$ & $2^{-}$ & $1^{0}$ & $2^{\underline{0}}$ & $1^{0}$ & $2^{0}$ \\
\hline & & & & & & ------ & & & & & & \\
\hline Pacovan & $23,4 \mathrm{~B}$ & $23,8 \mathrm{~A}$ & $1,4 \mathrm{~A}$ & $1,7 \mathrm{~A}$ & $19,3 \mathrm{C}$ & $24,2 \mathrm{~A}$ & $5,6 \mathrm{~B}$ & $17,1 \mathrm{~A}$ & $5,7 \mathrm{~A}$ & $6,9 \mathrm{~A}$ & $0,9 \mathrm{D}$ & $2,3 \mathrm{~A}$ \\
\hline Japira & $26,1 \mathrm{~A}$ & $25,0 \mathrm{~A}$ & $1,3 \mathrm{~A}$ & $1,6 \mathrm{~A}$ & $21,7 \mathrm{C}$ & $19,5 \mathrm{~B}$ & $7,1 \mathrm{~B}$ & $7,4 \mathrm{~B}$ & $3,6 \mathrm{C}$ & $3,9 \mathrm{C}$ & $1,8 \mathrm{~A}$ & $2,3 \mathrm{~A}$ \\
\hline PV42-129 & $24,8 \mathrm{~A}$ & $26,4 \mathrm{~A}$ & $1,2 \mathrm{~A}$ & $1,7 \mathrm{~A}$ & $18,7 \mathrm{C}$ & $19,0 \mathrm{~B}$ & $7,2 \mathrm{~B}$ & 7,9B & $4,1 \mathrm{~B}$ & $3,7 \mathrm{C}$ & $1,8 \mathrm{~A}$ & $1,7 \mathrm{~B}$ \\
\hline Preciosa & $25,0 \mathrm{~A}$ & $25,5 \mathrm{~A}$ & $1,5 \mathrm{~A}$ & $1,6 \mathrm{~A}$ & $23,7 \mathrm{~B}$ & $16,3 \mathrm{~B}$ & $6,1 \mathrm{~B}$ & $7,9 \mathrm{~B}$ & $3,6 \mathrm{C}$ & $3,8 \mathrm{C}$ & $1,9 \mathrm{~A}$ & $2,3 \mathrm{~A}$ \\
\hline Vitória & $24,5 \mathrm{~A}$ & $23,8 \mathrm{~A}$ & $1,4 \mathrm{~A}$ & $1,3 \mathrm{~A}$ & $18,3 \mathrm{C}$ & $14,3 \mathrm{~B}$ & $7,4 \mathrm{~B}$ & $9,6 \mathrm{~B}$ & $3,1 \mathrm{C}$ & $3,7 \mathrm{C}$ & $1,4 \mathrm{~B}$ & $1,3 \mathrm{~B}$ \\
\hline Pacovan Ken & $23,6 \mathrm{~B}$ & $24,6 \mathrm{~A}$ & $1,3 \mathrm{~A}$ & $1,5 \mathrm{~A}$ & $23,7 \mathrm{~B}$ & $17,0 \mathrm{~B}$ & $5,1 \mathrm{~B}$ & $6,5 \mathrm{~B}$ & $3,2 \mathrm{C}$ & $3,1 \mathrm{C}$ & $1,7 \mathrm{~A}$ & $1,3 \mathrm{~B}$ \\
\hline PV42-53 & $24,5 \mathrm{~A}$ & $24,9 \mathrm{~A}$ & $1,0 \mathrm{~A}$ & $1,6 \mathrm{~A}$ & $24,3 \mathrm{~B}$ & $13,7 \mathrm{~B}$ & $6,8 \mathrm{~B}$ & $9,7 \mathrm{~B}$ & $3,4 \mathrm{C}$ & $3,4 \mathrm{C}$ & $1,4 \mathrm{~B}$ & $1,3 \mathrm{~B}$ \\
\hline Prata Comum & $22,1 \mathrm{~B}$ & $26,0 \mathrm{~A}$ & $1,3 \mathrm{~A}$ & $1,7 \mathrm{~A}$ & $17,7 \mathrm{C}$ & $21,3 \mathrm{~A}$ & $7,5 \mathrm{~B}$ & $9,6 \mathrm{~B}$ & $5,7 \mathrm{~A}$ & $5,2 \mathrm{~B}$ & $0,8 \mathrm{D}$ & $2,6 \mathrm{~A}$ \\
\hline Prata-Caprichosa & $23,9 \mathrm{~B}$ & $26,5 \mathrm{~A}$ & $1,4 \mathrm{~A}$ & $1,8 \mathrm{~A}$ & $20,0 \mathrm{C}$ & $22,0 \mathrm{~A}$ & $6,0 \mathrm{~B}$ & $6,7 \mathrm{~B}$ & $4,3 \mathrm{~B}$ & $4,0 \mathrm{C}$ & $1,2 \mathrm{C}$ & $2,1 \mathrm{~A}$ \\
\hline Prata-Anã & $22,8 \mathrm{~B}$ & $26,4 \mathrm{~A}$ & $1,2 \mathrm{~A}$ & $1,6 \mathrm{~A}$ & $16,3 \mathrm{C}$ & $25,3 \mathrm{~A}$ & $8,3 \mathrm{~A}$ & $8,0 \mathrm{~B}$ & $5,1 \mathrm{~A}$ & $7,5 \mathrm{~A}$ & $0,8 \mathrm{D}$ & $2,3 \mathrm{~A}$ \\
\hline Pioneira & $22,3 \mathrm{~B}$ & $28,5 \mathrm{~A}$ & $1,4 \mathrm{~A}$ & $1,7 \mathrm{~A}$ & $17,7 \mathrm{C}$ & $25,8 \mathrm{~A}$ & $5,5 \mathrm{~B}$ & $9,6 \mathrm{~B}$ & $3,8 \mathrm{C}$ & $5,3 \mathrm{~B}$ & $1,1 \mathrm{C}$ & $2,7 \mathrm{~A}$ \\
\hline Maravilha & $23,0 \mathrm{~B}$ & $27,5 \mathrm{~A}$ & $1,5 \mathrm{~A}$ & $1,7 \mathrm{~A}$ & $23,0 \mathrm{~B}$ & $25,0 \mathrm{~A}$ & $6,9 \mathrm{~B}$ & $8,4 \mathrm{~B}$ & $3,9 \mathrm{C}$ & $4,2 \mathrm{C}$ & $1,2 \mathrm{C}$ & $1,8 \mathrm{~B}$ \\
\hline FHIA-18 & $23,4 \mathrm{~B}$ & $25,3 \mathrm{~A}$ & $1,3 \mathrm{~A}$ & $1,5 \mathrm{~A}$ & $24,7 \mathrm{~B}$ & $28,7 \mathrm{~A}$ & $6,7 \mathrm{~B}$ & $11,1 \mathrm{~B}$ & $4,5 \mathrm{~B}$ & $5,9 \mathrm{~B}$ & $1,2 \mathrm{C}$ & $2,3 \mathrm{~A}$ \\
\hline Prata-Graúda & $25,6 \mathrm{~A}$ & $25,7 \mathrm{~A}$ & $1,4 \mathrm{~A}$ & $1,5 \mathrm{~A}$ & $21,7 \mathrm{C}$ & $21,7 \mathrm{~A}$ & $8,0 \mathrm{~B}$ & $9,5 \mathrm{~B}$ & $4,6 \mathrm{~B}$ & $5,2 \mathrm{~B}$ & $1,2 \mathrm{C}$ & $2,6 \mathrm{~A}$ \\
\hline Calipso & $21,6 \mathrm{~B}$ & $24,8 \mathrm{~A}$ & $1,3 \mathrm{~A}$ & $1,7 \mathrm{~A}$ & $25,0 \mathrm{~B}$ & $27,0 \mathrm{~A}$ & $6,4 \mathrm{~B}$ & $6,8 \mathrm{~B}$ & $2,4 \mathrm{D}$ & $3,2 \mathrm{C}$ & $0,9 \mathrm{D}$ & $2,3 \mathrm{~A}$ \\
\hline Bucaneiro & $25,3 \mathrm{~A}$ & $24,1 \mathrm{~A}$ & $1,5 \mathrm{~A}$ & $1,7 \mathrm{~A}$ & $28,3 \mathrm{~A}$ & $26,8 \mathrm{~A}$ & $7,0 \mathrm{~B}$ & $8,4 \mathrm{~B}$ & $3,3 \mathrm{C}$ & $3,8 \mathrm{C}$ & $0,9 \mathrm{D}$ & $2,3 \mathrm{~A}$ \\
\hline Ambrosia & $23,1 \mathrm{~B}$ & $25,9 \mathrm{~A}$ & $1,2 \mathrm{~A}$ & $1,7 \mathrm{~A}$ & $29,3 \mathrm{~A}$ & $30,8 \mathrm{~A}$ & 4,8B & $7,5 \mathrm{~B}$ & $2,4 \mathrm{D}$ & $3,3 \mathrm{C}$ & $0,9 \mathrm{D}$ & $1,9 \mathrm{~B}$ \\
\hline Grande Naine & $25,0 \mathrm{~A}$ & $24,3 \mathrm{~A}$ & $1,6 \mathrm{~A}$ & $1,7 \mathrm{~A}$ & $22,3 \mathrm{~B}$ & $26,3 \mathrm{~A}$ & $11,1 \mathrm{~A}$ & $8,4 \mathrm{~B}$ & $3,8 \mathrm{C}$ & $3,6 \mathrm{C}$ & $0,9 \mathrm{D}$ & $2,7 \mathrm{~A}$ \\
\hline Thap Maeo & $23,0 \mathrm{~B}$ & $23,9 \mathrm{~A}$ & $1,2 \mathrm{~A}$ & $1,5 \mathrm{~A}$ & $18,3 \mathrm{C}$ & $15,7 \mathrm{~B}$ & $10,1 \mathrm{~A}$ & $13,3 \mathrm{~A}$ & $4,9 \mathrm{~B}$ & $5,0 \mathrm{~B}$ & $0,9 \mathrm{D}$ & $2,9 \mathrm{~A}$ \\
\hline Caipira & $26,3 \mathrm{~A}$ & $27,0 \mathrm{~A}$ & $1,4 \mathrm{~A}$ & $1,7 \mathrm{~A}$ & $24,7 \mathrm{~B}$ & $23,8 \mathrm{~A}$ & $7,3 \mathrm{~B}$ & $9,3 \mathrm{~B}$ & $3,0 \mathrm{D}$ & $3,4 \mathrm{C}$ & $1,0 \mathrm{C}$ & $2,2 \mathrm{~A}$ \\
\hline Nam & $24,8 \mathrm{~A}$ & $26,3 \mathrm{~A}$ & $1,6 \mathrm{~A}$ & $1,6 \mathrm{~A}$ & $30,3 \mathrm{~A}$ & $25,8 \mathrm{~A}$ & 9,9A & $9,3 \mathrm{~B}$ & $3,8 \mathrm{C}$ & $3,6 \mathrm{C}$ & $1,5 \mathrm{~B}$ & $1,5 \mathrm{~B}$ \\
\hline Tropical & $25,2 \mathrm{~A}$ & $27,5 \mathrm{~A}$ & $1,4 \mathrm{~A}$ & $1,6 \mathrm{~A}$ & $24,3 \mathrm{~B}$ & $23,7 \mathrm{~A}$ & $4,8 \mathrm{~B}$ & $6,6 \mathrm{~B}$ & $2,6 \mathrm{D}$ & $3,5 \mathrm{C}$ & $1,2 \mathrm{C}$ & $2,1 \mathrm{~A}$ \\
\hline Prata-Garantida & $25,5 \mathrm{~A}$ & $24,0 \mathrm{~A}$ & $1,3 \mathrm{~A}$ & $1,8 \mathrm{~A}$ & $25,0 \mathrm{~B}$ & $22,5 \mathrm{~A}$ & $6,1 \mathrm{~B}$ & $7,9 \mathrm{~B}$ & $3,4 \mathrm{C}$ & $4,1 \mathrm{C}$ & $1,1 \mathrm{C}$ & $2,3 \mathrm{~A}$ \\
\hline ST12-31 & $26,3 \mathrm{~A}$ & $26,2 \mathrm{~A}$ & $1,4 \mathrm{~A}$ & $1,7 \mathrm{~A}$ & $19,3 \mathrm{C}$ & $18,4 \mathrm{~B}$ & $8,0 \mathrm{~B}$ & $7,5 \mathrm{~B}$ & $4,5 \mathrm{~B}$ & $4,1 \mathrm{C}$ & $1,2 \mathrm{C}$ & $2,1 \mathrm{~A}$ \\
\hline Média & 24,2 & 25,6 & 1,4 & 1,6 & 22,4 & 22,3 & 7,1 & 9,0 & 3,9 & 4,3 & 1,2 & 2,1 \\
\hline CV (\%) & 6,6 & 7,1 & 11,3 & 9,9 & 12,1 & 17,5 & 22,5 & 29,5 & 16,1 & 19,4 & 17,2 & 17,7 \\
\hline
\end{tabular}

${ }^{1}$ Médias seguidas pela mesma letra, na coluna, pertencem ao mesmo agrupamento, pelo teste de Scott \& Knott, a 5\% de probabilidade.

híbridos (Tabela 1) com os teores de $\mathrm{N}$ propostos por Silva et al. (2002) para o norte de Minas Gerais (25,0-29,0 $\mathrm{g} \mathrm{kg}^{-1}$ ), observou-se que o teor nos genótipos avaliados está dentro da faixa no segundo ciclo (média de 26,7 $\left.\mathrm{g} \mathrm{kg}^{-1}\right)$ e apenas para 'Prata-Graúda' $\left(25,6 \mathrm{~g} \mathrm{~kg}^{-1}\right)$ no primeiro ciclo (Tabela 3). Para 'Pacovan' e seus híbridos (Tabela 1), nos dois ciclos, os teores de $\mathrm{N}$ estão dentro da faixa definida por Borges \& Caldas (2004), com médias de 25,1 e 23,5 $\mathrm{g} \mathrm{kg}^{-1}$, no primeiro ciclo, e de 24,9 $\mathrm{g} \mathrm{kg}^{-1}$, no segundo ciclo (Tabela 3).

Para o $\mathrm{P}$, o teor médio foi menor no primeiro ciclo $\left(1,4 \mathrm{~g} \mathrm{~kg}^{-1}\right)$, porém, no segundo ciclo, situou-se na faixa estabelecida por IFA(1992) e Silva et al. (2002).

Comparando o teor de K da 'Prata-Anã' e seus híbridos, apenas a FHIA-18, no segundo ciclo $\left(28,7 \mathrm{~g} \mathrm{~kg}^{-1}\right)$, apresentou teor dentro da faixa estabelecida por Silva et al. (2002). O mesmo ocorreu para a 'Pacovan'e seus híbridos (médias de 23,9 e 19,6, no 1º ciclo, e $16,3 \mathrm{~g} \mathrm{~kg}^{-1}$, no $2^{\circ}$ ciclo), cujos valores não se encontraram na faixa estabelecida por Borges \& Caldas (2004) (Tabela 3). Isso indica que as condições ambientais influenciam nos teores foliares, ou seja, os teores padrões de referência não têm aplicação universal, necessitando ser adaptados para as condições locais, embora alguma extrapolação possa ser feita (Bataglia \& Dechen, 1986). Vale ressaltar que o K é o nutriente mais absorvido pela bananeira, importante como ativador enzimático e participa de processos como abertura e fechamento de estômatos, fotossíntese, transporte de carboidratos e respiração (Malavolta et al., 1989).

Quanto ao Ca, o teor médio nos híbridos de 'Prata-Anã', no primeiro ciclo $\left(6,8 \mathrm{~g} \mathrm{~kg}^{-1}\right)$, está dentro dos teores adequados, estando acima no segundo ciclo $\left(9,3 \mathrm{~g} \mathrm{~kg}^{-1}\right)$, segundo a faixa estabelecida por Silva et al. (2002). Para 'Pacovan' (17,1 $\mathrm{g} \mathrm{kg}^{-1}$ ) e seus híbridos (média de $8,2 \mathrm{~g} \mathrm{~kg}^{-1}$ ), no segundo ciclo, os teores estão acima da faixa estabelecida por Borges \& Caldas (2004) (Tabela 3). No primeiro ciclo, tanto para 'Pacovan' (5,6 $\left.\mathrm{g} \mathrm{kg}^{-1}\right)$ quanto para 'Pacovan Ken' $(5,1 \mathrm{~g} \mathrm{~kg}$ ${ }^{1}$ ), os teores ficaram abaixo dos valores de Borges \& Caldas (2004); contudo, a média $\left(6,5 \mathrm{~g} \mathrm{~kg}^{-1}\right)$ está dentro da faixa. O Ca tem função estrutural, sendo constituinte de pectatos de $\mathrm{Ca}$ da lamela média das células, participando dos processos de absorção iônica (Malavolta et al., 1989).

$\mathrm{O} \mathrm{Mg}$ apresentou comportamento semelhante ao $\mathrm{Ca}$, apesar do menor teor médio encontrado nas folhas da bananeira (Tabela 3). Os teores de Mg na 'Pioneira' e 'Maravilha' (média de 3,9 $\mathrm{g} \mathrm{kg}^{-1}$ ), no primeiro ciclo, estão na faixa estabelecida por Silva et al. (2002); enquanto FHIA18 e 'Prata-Graúda' (média de 4,6 $\mathrm{g} \mathrm{kg}^{-1}$ ) e 'Prata-Anã' mais altos (Tabela 3). No primeiro ciclo, o teor médio de Mg dos genótipos Japira, Preciosa, Vitória, Pacovan Ken e PV42-53 foi de 3,4 $\mathrm{g} \mathrm{kg}^{-1}$; enquanto, no segundo ciclo, o teor médio dos híbridos da 'Pacovan' (3,6 $\left.\mathrm{g} \mathrm{kg}^{-1}\right)$ situou-se na faixa estabelecida por Borges \& Caldas (2004). O Mg é ativador de enzimas e participa nos processos de absorção iônica, na fotossíntese e na respiração (Malavolta et al., 1989).

O S, no primeiro ciclo, apresentou teores abaixo dos estabelecidos por Silva et al. (2002) (1,7-2,0 $\left.\mathrm{g} \mathrm{kg}^{-1}\right)$ para 'Prata-Anã $\left(0,8 \mathrm{~g} \mathrm{~kg}^{-1}\right)$ e seus híbridos (média de $\left.1,2 \mathrm{~g} \mathrm{~kg}^{-1}\right)$; no segundo ciclo, os teores foram adequados para 'Maravilha' e ligeiramente superiores para os demais genótipos (média de 2,5 $\mathrm{g} \mathrm{kg}^{-1}$ ) (Tabela 3). Para 'Pacovan' (0,9 $\left.\mathrm{g} \mathrm{kg}^{-1}\right)$, 'Vitória' e PV42-53 (1,4 $\left.\mathrm{g} \mathrm{kg}^{-1}\right)$, no primeiro ciclo, e 'Vitória', 'Pacovan Ken', PV42-53 e PV42-129, no segundo ciclo (Tabela 3), o teor médio (1,3 $\left.\mathrm{g} \mathrm{kg}^{-1}\right)$ situou-se abaixo da faixa de 1,7-1,9 $\mathrm{g} \mathrm{kg}^{-1}$ (Borges \& Caldas, 2004). Vale ressaltar que o $\mathrm{S}$ apresenta funções estruturais na planta como componente de aminoácidos, proteínas, vitaminas e coenzinas (Malavolta et al., 1989).

Quanto aos micronutrientes, o $\mathrm{Cl}$, apesar de ser um micronutriente, é expresso em $\mathrm{g} \mathrm{kg}^{-1}$, devido ao cloreto de potássio utilizado como fonte de $\mathrm{K}$ e que contém em torno de $300 \mathrm{~g} \mathrm{de} \mathrm{Cl} \mathrm{kg}^{-1}$ do fertilizante. No primeiro ciclo, foram observados três agrupamentos (médias de 24,2; 20,7 e 15,4 $\mathrm{g} \mathrm{kg}^{-1}$ ) e apenas um no segundo ciclo (Tabela 4). No primeiro ciclo, os genótipos originados da 'Prata-Anã' - 'Maravilha' e FHIA-18 (média de 20,2 $\mathrm{g} \mathrm{kg}^{-1}$ ) e da 'Pacovan' - 'Vitória' 
TABELA 4 - Teores foliares de micronutrientes em 24 genótipos de bananeira, no primeiro (1ํํ) e segundo ( $\left.2^{\circ}\right)$ ciclos de produção, nos anos de 1999-2000, Cruz das Almas-BA.

\begin{tabular}{|c|c|c|c|c|c|c|c|c|c|c|c|c|}
\hline \multirow{3}{*}{ Genótipos } & \multicolumn{2}{|c|}{ Cl } & \multicolumn{2}{|c|}{ B } & \multicolumn{2}{|c|}{$\mathbf{C u}$} & \multicolumn{2}{|c|}{ Fe } & \multicolumn{2}{|c|}{ Mn } & \multicolumn{2}{|c|}{$\mathbf{Z n}$} \\
\hline & $\mathbf{1}^{\mathbf{0}}$ & $2^{\mathbf{0}}$ & 10 & $2^{\underline{0}}$ & 1 & $2^{\mathbf{0}}$ & 1 1 & $2^{\underline{0}}$ & $\mathbf{1}^{\mathbf{0}}$ & $2^{\mathbf{0}}$ & $1 \mathbf{0}$ & $2^{\mathbf{0}}$ \\
\hline & \multicolumn{2}{|c|}{--- g. $\mathbf{k g}^{-1}---$} & \multicolumn{10}{|c|}{ 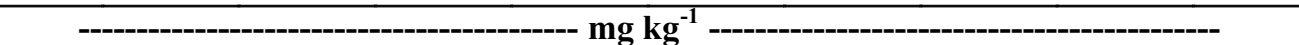 } \\
\hline Pacovan & $17,3 \mathrm{C}$ & $14,2 \mathrm{~A}$ & $51,3 \mathrm{C}$ & $22,0 \mathrm{~A}$ & $9,7 \mathrm{~A}$ & $7,0 \mathrm{C}$ & $131 \mathrm{~B}$ & $97 \mathrm{~A}$ & $446 \mathrm{~A}$ & $353 \mathrm{~B}$ & $39,3 \mathrm{~B}$ & $18,3 \mathrm{~A}$ \\
\hline Japira & $14,7 \mathrm{C}$ & $12,5 \mathrm{~A}$ & $26,0 \mathrm{D}$ & $27,5 \mathrm{~A}$ & $2,3 \mathrm{D}$ & $6,0 \mathrm{C}$ & $101 B$ & $71 \mathrm{~A}$ & $383 \mathrm{~A}$ & $491 \mathrm{~A}$ & $17,3 \mathrm{C}$ & $13,5 \mathrm{D}$ \\
\hline PV42-129 & $15,7 \mathrm{C}$ & $12,1 \mathrm{~A}$ & $28,3 \mathrm{D}$ & $21,7 \mathrm{~A}$ & $2,0 \mathrm{D}$ & $7,3 \mathrm{C}$ & $115 \mathrm{~B}$ & $82 \mathrm{~A}$ & $574 \mathrm{~A}$ & 245B & $17,0 \mathrm{C}$ & $14,3 \mathrm{D}$ \\
\hline Preciosa & $15,7 \mathrm{C}$ & $14,2 \mathrm{~A}$ & $26,0 \mathrm{D}$ & $18,7 \mathrm{~A}$ & $2,3 \mathrm{D}$ & $7,0 \mathrm{C}$ & $95 \mathrm{~B}$ & $120 \mathrm{~A}$ & $370 \mathrm{~A}$ & $503 \mathrm{~A}$ & $19,0 \mathrm{C}$ & $13,7 \mathrm{D}$ \\
\hline Vitória & $22,0 \mathrm{~B}$ & $13,8 \mathrm{~A}$ & $28,3 \mathrm{D}$ & $19,0 \mathrm{~A}$ & $2,0 \mathrm{D}$ & $8,0 \mathrm{C}$ & $80 \mathrm{~B}$ & $84 \mathrm{~A}$ & $240 B$ & 519A & $14,0 \mathrm{C}$ & $12,5 \mathrm{D}$ \\
\hline Pacovan Ken & $15,0 \mathrm{C}$ & $12,1 \mathrm{~A}$ & $26,0 \mathrm{D}$ & $21,7 \mathrm{~A}$ & $2,3 \mathrm{D}$ & $6,7 \mathrm{C}$ & $102 B$ & $104 \mathrm{~A}$ & $320 \mathrm{~A}$ & $519 A$ & $16,3 \mathrm{C}$ & $12,3 \mathrm{D}$ \\
\hline PV42-53 & $20,0 \mathrm{~B}$ & $13,3 \mathrm{~A}$ & $25,7 \mathrm{D}$ & $22,3 \mathrm{~A}$ & $2,0 \mathrm{D}$ & $7,0 \mathrm{C}$ & 47B & $117 \mathrm{~A}$ & $124 B$ & 399A & $14,7 \mathrm{C}$ & $13,7 \mathrm{D}$ \\
\hline Prata Comum & $20,3 \mathrm{~B}$ & $15,0 \mathrm{~A}$ & $51,0 \mathrm{C}$ & $13,7 \mathrm{~A}$ & $6,3 \mathrm{C}$ & $7,3 \mathrm{C}$ & 109B & $86 \mathrm{~A}$ & $260 B$ & 229B & $38,0 \mathrm{~B}$ & $17,3 \mathrm{~B}$ \\
\hline Prata-Caprichosa & $15,3 \mathrm{C}$ & $12,5 \mathrm{~A}$ & $72,0 \mathrm{~B}$ & $36,0 \mathrm{~A}$ & $5,0 \mathrm{C}$ & $7,0 \mathrm{C}$ & $158 \mathrm{~A}$ & $56 \mathrm{~A}$ & $340 \mathrm{~A}$ & 222B & $21,0 \mathrm{C}$ & $14,0 \mathrm{D}$ \\
\hline Prata-Anã & $23,7 \mathrm{~A}$ & $13,8 \mathrm{~A}$ & $24,7 \mathrm{D}$ & $20,5 \mathrm{~A}$ & $6,0 \mathrm{C}$ & $6,5 \mathrm{C}$ & $184 \mathrm{~A}$ & $94 \mathrm{~A}$ & $454 \mathrm{~A}$ & 319B & $17,7 \mathrm{C}$ & $18,5 \mathrm{~A}$ \\
\hline Pioneira & $17,7 \mathrm{C}$ & $13,3 \mathrm{~A}$ & $89,0 \mathrm{~A}$ & $20,7 \mathrm{~A}$ & $5,3 \mathrm{C}$ & $7,3 \mathrm{C}$ & $111 B$ & $105 \mathrm{~A}$ & 492A & $483 \mathrm{~A}$ & $36,3 \mathrm{~B}$ & $17,0 \mathrm{~B}$ \\
\hline Maravilha & $21,0 \mathrm{~B}$ & $15,8 \mathrm{~A}$ & $41,7 \mathrm{C}$ & $15,3 \mathrm{~A}$ & $5,7 \mathrm{C}$ & $12,0 \mathrm{~B}$ & $150 \mathrm{~A}$ & $81 \mathrm{~A}$ & 334A & $512 \mathrm{~A}$ & $59,3 \mathrm{~A}$ & $17,0 \mathrm{~B}$ \\
\hline FHIA-18 & $19,3 \mathrm{~B}$ & $10,4 \mathrm{~A}$ & $95,7 \mathrm{~A}$ & $21,3 \mathrm{~A}$ & $6,0 \mathrm{C}$ & $14,0 \mathrm{~A}$ & $186 \mathrm{~A}$ & $76 \mathrm{~A}$ & $500 \mathrm{~A}$ & $572 \mathrm{~A}$ & $33,3 \mathrm{~B}$ & $16,3 \mathrm{~B}$ \\
\hline Prata-Graúda & $15,3 \mathrm{C}$ & $13,8 \mathrm{~A}$ & $25,7 \mathrm{D}$ & $24,0 \mathrm{~A}$ & $5,0 \mathrm{C}$ & $5,3 \mathrm{C}$ & $159 \mathrm{~A}$ & $89 \mathrm{~A}$ & $178 \mathrm{~B}$ & $158 \mathrm{~B}$ & $20,3 \mathrm{C}$ & $13,0 \mathrm{D}$ \\
\hline Calipso & $21,0 \mathrm{~B}$ & $12,5 \mathrm{~A}$ & $50,3 \mathrm{C}$ & $17,0 \mathrm{~A}$ & $7,0 \mathrm{C}$ & $9,0 \mathrm{C}$ & $182 \mathrm{~A}$ & $103 \mathrm{~A}$ & $437 \mathrm{~A}$ & 468A & $57,0 \mathrm{~A}$ & $20,5 \mathrm{~A}$ \\
\hline Bucaneiro & $24,7 \mathrm{~A}$ & $15,0 \mathrm{~A}$ & $55,7 \mathrm{C}$ & $16,7 \mathrm{~A}$ & $5,3 \mathrm{C}$ & $8,7 \mathrm{C}$ & 99B & $86 \mathrm{~A}$ & 88B & $513 \mathrm{~A}$ & $35,7 \mathrm{~B}$ & $19,7 \mathrm{~A}$ \\
\hline Ambrosia & $14,3 \mathrm{C}$ & $15,0 \mathrm{~A}$ & $36,0 \mathrm{D}$ & $17,0 \mathrm{~A}$ & $6,7 \mathrm{C}$ & $11,7 \mathrm{~B}$ & $85 B$ & $90 \mathrm{~A}$ & $43 B$ & $224 B$ & $36,7 \mathrm{~B}$ & $18,0 \mathrm{~B}$ \\
\hline Grande Naine & $21,0 \mathrm{~B}$ & $12,9 \mathrm{~A}$ & $27,3 \mathrm{D}$ & $19,0 \mathrm{~A}$ & $5,3 \mathrm{C}$ & $7,7 \mathrm{C}$ & $212 \mathrm{~A}$ & $78 \mathrm{~A}$ & $158 \mathrm{~B}$ & 203B & $34,0 \mathrm{~B}$ & $19,0 \mathrm{~A}$ \\
\hline Thap Maeo & $16,0 \mathrm{C}$ & $12,5 \mathrm{~A}$ & $38,7 \mathrm{C}$ & $22,3 \mathrm{~A}$ & $6,7 \mathrm{C}$ & $5,3 \mathrm{C}$ & $100 \mathrm{~B}$ & $76 \mathrm{~A}$ & $116 \mathrm{~B}$ & 259B & $2,3 \mathrm{C}$ & $15,3 \mathrm{C}$ \\
\hline Caipira & $13,0 \mathrm{C}$ & $11,7 \mathrm{~A}$ & $34,7 \mathrm{D}$ & $20,0 \mathrm{~A}$ & $6,3 \mathrm{C}$ & $6,7 \mathrm{C}$ & $116 \mathrm{~B}$ & $91 \mathrm{~A}$ & $118 \mathrm{~B}$ & $185 \mathrm{~B}$ & $47,0 \mathrm{~A}$ & $16,0 \mathrm{C}$ \\
\hline Nam & $21,0 \mathrm{~B}$ & $15,0 \mathrm{~A}$ & $35,0 \mathrm{D}$ & $29,3 \mathrm{~A}$ & $8,0 \mathrm{~B}$ & $7,7 \mathrm{C}$ & $128 \mathrm{~B}$ & $68 \mathrm{~A}$ & $183 \mathrm{~B}$ & $170 \mathrm{~B}$ & $28,0 \mathrm{C}$ & $15,7 \mathrm{C}$ \\
\hline Tropical & $14,0 \mathrm{C}$ & $10,8 \mathrm{~A}$ & $25,3 \mathrm{D}$ & $27,7 \mathrm{~A}$ & $7,3 \mathrm{~B}$ & $7,0 \mathrm{C}$ & $125 \mathrm{~B}$ & $57 \mathrm{~A}$ & 272B & $132 \mathrm{~B}$ & $16,3 \mathrm{C}$ & $15,0 \mathrm{C}$ \\
\hline Prata-Garantida & $15,0 \mathrm{C}$ & $11,7 \mathrm{~A}$ & $20,3 \mathrm{D}$ & $30,3 \mathrm{~A}$ & $3,0 \mathrm{D}$ & $7,0 \mathrm{C}$ & $123 B$ & $78 \mathrm{~A}$ & $183 \mathrm{~B}$ & $154 \mathrm{~B}$ & $17,7 \mathrm{C}$ & $15,7 \mathrm{C}$ \\
\hline ST12-31 & $17,0 \mathrm{C}$ & $13,1 \mathrm{~A}$ & $27,7 \mathrm{D}$ & $35,0 \mathrm{~A}$ & $2,0 \mathrm{D}$ & $7,5 \mathrm{C}$ & $89 B$ & $64 \mathrm{~A}$ & $267 \mathrm{~B}$ & $224 B$ & $16,7 \mathrm{C}$ & $13,8 \mathrm{D}$ \\
\hline Média & 17,9 & 13,2 & 40,1 & 22,5 & 5,0 & 7,8 & 124 & 86 & 285 & 329 & 27,3 & 15,8 \\
\hline CV (\%) & 10,2 & 14,9 & 23,5 & 37,6 & 23,4 & 14,6 & 31,1 & 22,7 & 48,0 & 33,0 & 42,3 & 7,1 \\
\hline
\end{tabular}

${ }^{1}$ Médias seguidas pela mesma letra, na coluna, pertencem ao mesmo grupo, pelo teste de Scott \& Knott, a 5\% de probabilidade.

e PV42-53 (média de 21,0 $\mathrm{g} \mathrm{kg}^{-1}$ ) (Tabela 4) estão acima da faixa estabelecida por IFA (1992). Vale ressaltar que, apesar de a bananeira tolerar alta concentração de $\mathrm{Cl}$, teores acima de $20 \mathrm{~g} \mathrm{~kg}^{-1}$ são considerados altos e $35 \mathrm{~g} \mathrm{de} \mathrm{Cl} \mathrm{kg}^{-1}$ tóxicos (IFA, 1992). A toxidez de Cl pode levar à produção de frutos magros, sem condições de comercialização. Neste caso, devem-se utilizar outras fontes de K.

Os micronutrientes $\mathrm{B}$ e $\mathrm{Zn}$ são aqueles cujas deficiências são mais comuns na bananeira. Para $\mathrm{B}$, no primeiro ciclo, foram observados quatro agrupamentos (médias de 92,4; 72,0; 48,1 e 27,8 $\mathrm{mg} \mathrm{kg}^{-1}$ ); já no segundo ciclo, apenas um agrupamento (Tabela 4). No primeiro ciclo, foram encontrados teores de $\mathrm{B}$ muito altos nos híbridos da 'Prata-Anã', notadamente na FHIA-18 e 'Pioneira' (média de 92,4 $\mathrm{mg} \mathrm{kg}{ }^{-1}$ ), sem contudo ter uma explicação para o ocorrido (Tabela 4). Segundo IFA (1992), teores na faixa de 30 a $100 \mathrm{mg} \mathrm{kg}^{-1}$ são considerados tóxicos para a bananeira. Para 'Prata-Anã' e seus híbridos (média de 20,4 $\mathrm{mg} \mathrm{kg}^{-1}$ ), no segundo ciclo, os teores de B situaram-se na faixa estabelecida por Silva et al. (2002). Para 'Pacovan' (51,3 $\mathrm{mg} \mathrm{kg}^{-1}$ ) e seus híbridos (média de $26,7 \mathrm{mg} \mathrm{kg}^{-1}$ ), no primeiro ciclo, e também no segundo ciclo (média da Pacovan e híbridos de $21,8 \mathrm{mg} \mathrm{kg}^{-1}$ ), os teores apresentaram-se acima dos estabelecidos por Borges \& Caldas (2004) (Tabela 4).

Para o micronutriente Zn, observa-se na Tabela 4 a formação de três agrupamentos no primeiro ciclo (médias de 54,4;36,2 e 17,0 mg $\mathrm{kg}^{-1}$ ) e quatro no segundo ciclo (médias de 19,2;17,1; 15,5 e 13,4 mg $\mathrm{kg}^{-1}$ ). Os teores de $\mathrm{Zn}$ apresentaram-se no primeiro ciclo superiores aos propostos por Silva et al. (2002) para 'Pioneira' e FHIA-18 (34,8 mg kg-1) e 'Maravilha' (59,3 mg kg-1) e dentro da faixa, exceto para 'Prata-Graúda', no segundo ciclo $\left(13,0 \mathrm{mg} \mathrm{kg}^{-1}\right)$. Para os híbridos da 'Pacovan', os teores estão adequados no segundo ciclo (média de $13,3 \mathrm{mg} \mathrm{kg}^{-1}$ ), segundo a faixa de Borges \& Caldas (2004). No primeiro ciclo, os teores estão mais elevados (média de $16,4 \mathrm{mg} \mathrm{kg}^{-1}$ ), notadamente para a 'Pacovan' (Tabela 4).

Os teores de $\mathrm{Cu}$ nos genótipos formaram quatro agrupamentos no primeiro ciclo $\left(9,7 ; 7,7 ; 5,9\right.$ e $\left.2,2 \mathrm{mg} \mathrm{kg}^{-1}\right)$ e três no segundo ciclo $\left(14,0 ; 11,9\right.$ e 7,1 $\left.\mathrm{mg} \mathrm{kg}^{-1}\right)$ (Tabela 4). Os teores estão acima dos estabelecidos por Silva et al. (2002) para 'Prata-Anã' (2,6$\left.8,8 \mathrm{mg} \mathrm{kg}^{-1}\right)$, nos genótipos Maravilha (12 $\left.\mathrm{mg} \mathrm{kg}^{-1}\right)$ e FHIA-18 (14,0 mg $\mathrm{kg}^{-1}$ ), no segundo ciclo. Para os híbridos da 'Pacovan', os teores encontrados no primeiro ciclo $\left(2,2 \mathrm{mg} \mathrm{kg}^{-1}\right)$ estão bem abaixo da faixa estabelecida por Borges \& Caldas (2004), 6-7 mg kg-1 enquanto, no segundo ciclo, houve um incremento do teor médio para $8,2 \mathrm{mg} \mathrm{kg}^{-1}$ (Tabela 4).

Ferro e manganês não têm apresentado, na maioria das vezes, deficiências nas bananeiras, em razão dos altos teores desses nutrientes nos solos brasileiros. Os teores de ferro estão classificados

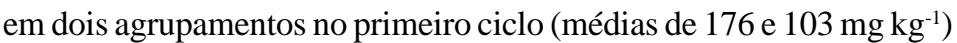
e apenas um no segundo ciclo (Tabela 4). No primeiro ciclo, os teores situaram-se acima dos valores estabelecidos por Silva et al. (2002) para 'Prata-Anã', exceto para 'Pioneira' (Tabela 4). De acordo com a faixa adequada de Fe (Borges \& Caldas, 2004), no primeiro ciclo, os genótipos apresentaram o teor médio $12 \%$ superior ao teor máximo estabelecido (Tabela 4).

No caso do Mn, observaram-se dois agrupamentos no primeiro (média de 423 e $172 \mathrm{mg} \mathrm{kg}^{-1}$ ) e segundo ciclos (498 e $220 \mathrm{mg}$ $\left.\mathrm{kg}^{-1}\right)$. Apenas o teor encontrado nas folhas da 'Prata-Graúda', no segundo ciclo $\left(158 \mathrm{mg} \mathrm{kg}^{-1}\right)$, foi inferior ao proposto por Silva et al. (2002) para 'Prata-Anã' (173-630 mg kg-1). Teores médios mais elevados, no valor de $419 \mathrm{mg} \mathrm{kg}^{-1}$, foram encontrados na 'Pacovan', 'Japira', PV42-129, 'Preciosa' e 'Pacovan Ken, no primeiro ciclo, e na 'Japira', 'Preciosa', 'Vitória', 'Pacovan Ken'e e PV42-53 (média de 399 $\mathrm{mg} \mathrm{kg}^{-1}$ ), no segundo ciclo (Tabela 4). Além disso, teores mais baixos (média de $182 \mathrm{mg} \mathrm{kg}^{-1}$ ) no genótipo 'Vitória' e PV42-53 (124 mg kg-1), no primeiro ciclo, e 'Pacovan' e PV42-129 (299 $\left.\mathrm{mg} \mathrm{kg}^{-1}\right)$ segundo a faixa proposta por Borges \& Caldas (2004), para 'Pacovan' (Tabela 4).

Apesar das características e origem genética semelhante, o agrupamento genômico não permitiu definir faixas dos teores de macro e micronutrientes de acordo com o grupo genômico. 


\section{CONCLUSÕES}

1) Existe variação nos teores foliares de nutrientes entre plantas do mesmo grupo genômico.

2) Foram observadas variações nos teores de nutrientes foliares no primeiro e segundo ciclos de produção, com teores médios mais elevados no segundo ciclo para $\mathrm{N}, \mathrm{P}, \mathrm{Ca}, \mathrm{Mg}, \mathrm{S}, \mathrm{Cu}$ e $\mathrm{Mn}$ e mais baixos para $\mathrm{K}, \mathrm{Cl}, \mathrm{B}, \mathrm{Fe}$ e $\mathrm{Zn}$.

3) O nitrogênio e o potássio foram os macronutrientes com teores mais elevados nas folhas, independentemente do genótipo.

4) O cloro, o manganês e o ferro foram os micronutrientes com teores mais elevados nas folhas.

\section{REFERÊNCIAS}

BATAGLIA, O.C.; FURLANI, A.M.; TEIXEIRA, J.P.F.; GALLO, J.R. Métodos de análise química de planta. Campinas-SP: Instituto Agronômico, 1983.48p. (Boletim Técnico, 78).

BATAGLIA, O.C.; DECHEN, A.R. Critérios alternativos para diagnose foliar. In: SIMPÓSIO AVANÇADODEQUÍMICAEFERTILIDADE DO SOLO, 1., 1986, Piracicaba-SP. Anais... Piracicaba-SP: Fundação Cargill, 1986. p.115-136.

BORGES, A.L.; CALDAS, R.C. Teores de nutrientes nas folhas de bananeira, cv. Pacovan, sob irrigação. Ciência e Agrotecnologia, Lavras, v.28, n.5, p.1099-1106, 2004.

FARIA, N.G. Absorção de nutrientes por variedades e híbridos promissores de bananeira. 1997. Dissertação (Mestrado) Universidade Federal da Bahia, Cruz das Almas, 1997. 66p.

IFA - International Fertilizer Industry Association (Paris). World fertilizer use manual. Limburgerhof: BASF. Agricultural Research Station, 1992.631p.
MALAVOLTA, E.; VITTI, G.C.; OLIVEIRA, S.A. de. Avaliação do estado nutricional das plantas: princípios e aplicações. Piracicaba: Associação Brasileira para Pesquisa da Potassa e do Fosfato, 1989.201p.

MARTIN-PRÉVEL, P. Echantillonnage du bananier pour l'analyse foliare: conséquences des différences de techniques. Fruits, Paris, v.32, p.151-166, 1977.

MUNSON, R.D.; NELSON, W.L. Principles and practices in plant analysis. In: WALSH, L.M.; BEATON, J.D. (Ed.). Soil testing and plant analysis. Madison: Soil Science Society of America, 1973. p.223-248.

NEVES, R.L.L.; FERREIRA, F.F.H.; MACIEL, R.F.P.; FROTA, J.N.E. Extração de nutrientes em bananeira (Musa sp) cv. Pacovan. Ciência Agronômica, Fortaleza, v.22, n.1/2, p.115-120, 1991.

SAEG - Sistema para Análises Estatísticas. v.9.0. Viçosa: Fundação Arthur Bernardes. Viçosa, UFV-MG. Disponível em: <http:// www.saeg.com.br>. Acesso em: 29 ago. 2005.

SAS INSTITUTE. SAS/STAT user's guide Cary NC: Sas Institute, 2005. v.1-3.

SCOTT, A.J.; KNOTT, M.A. A cluster analysis method for grouping means in the analysis of variance. Biometrics, Washington, v.30, n.3, p.507-512, 1974.

SILVA, J.T.A. da; BORGES, A.L.; DIAS, M.S.C.; COSTA, E.L. da; PRUDÊNCIO, J.M. Diagnóstico nutricional da bananeira 'Prata Anã' para o Norte de Minas Gerais. Belo Horizonte: EPAMIG, 2002. 16p. (Boletim Técnico, 70).

SOUZA, L. da S.; SOUZA, L.D. Caracterização físico-hídrica de solos da área do Centro Nacional de Pesquisa de Mandioca e Fruticultura Tropical. Cruz das Almas: Embrapa Mandioca e Fruticultura, 2001. 56p. (Boletim de Pesquisa e Desenvolvimento, 20).

WALSH, L.M.; BEATON, J.D. (Ed). Soil testing and plant analysis. Madison: Soil Science Society of America, 1973. 491p. 